POS $\quad$ PROCEEDINGS

\title{
CNO Abundances in Metal-Poor Stars
}

\section{Catherine R. Kennedy*}

Michigan State University/JINA

E-mail: kenne257@msu.edu

Timothy C. Beers

Michigan State University/JINA

E-mail: beers @pa.msu.edu

\section{Birgitta Nordström}

University of Copenhagen, Niels Bohr Institute

E-mail: birgitta@nbi.ku.dk

\section{Vinicius M. Placco, Silvia Rossi}

IAG, University of São Paulo

E-mail: vmplaccolastro.iag.usp.br, rossi@astro.iag.usp.br

\section{Thirupathi Sivarani}

Indian Institute of Astrophysics

E-mail: sivarani@iiap.res.in

Carbon, nitrogen, and oxygen produced in the early universe come from a variety of possible astrophysical sites. Among these are early supernovae, winds of massive, rapidly-rotating, mega metal-poor stars, and intermediate mass AGB stars. Large-scale surveys such as the HK Survey of Beers and colleagues and the Hamburg/ESO Survey of Christlieb and colleagues have allowed for the identification of numerous metal-poor stars in the Galactic halo. Follow-up observations of these metal-poor stars is necessary to determine $\mathrm{CNO}$ abundances. Techniques have been developed such that $[\mathrm{C} / \mathrm{Fe}],[\mathrm{N} / \mathrm{Fe}]$, and $[\mathrm{O} / \mathrm{Fe}]$ can be estimated with considerable accuracy using medium-resolution observations alone. We present estimates of these species for a number of metal-poor stars based on analysis of near-UV, optical, and near-IR spectra. The data come from several different instruments on southern-hemisphere telescopes, including the Goodman HTS and OSIRIS on SOAR, GMOS-S on Gemini, and XSHOOTER on VLT. In this way, we present some of the first metal-poor stars with a full complement of $\mathrm{CNO}$ abundances based solely on the analysis of medium-resolution spectra.

11th Symposium on Nuclei in the Cosmos

19-23 July 2010

Heidelberg, Germany.

\footnotetext{
* Speaker.
} 


\section{Introduction}

Carbon, nitrogen, and oxygen are often found to be over-abundant in metal-poor stars. By studying the abundance patterns of such stars, one is able to constrain the properties of galactic chemical evolution (GCE) as well as the overall formation of the Milky Way. Carbon-enhanced metal-poor stars exist in several different varieties (Beers \& Christlieb 2005). CEMP-s stars are those enhanced in s-process elements, while CEMP-r have enhancements of r-process nuclei. Even more peculiar are the CEMP-no stars, which commonly exhibit CNO over-abundances with no neutron-capture-element enhancements. The possible sites of production of these objects include mass-transfer from a nearby AGB companion (the likely source of CEMP-s stars) and mass-loss by rapidly-rotating, mega metal-poor stars with $[\mathrm{Fe} / \mathrm{H}]<-6.0$ (Hirschi et al. 2006; Meynet et al. 2006), a possible source of CEMP-no stars. By studying a large number of metal-poor stars, one can begin to understand the origins of the elements, and significant progress can be made by utilizing medium-resolution spectroscopy to estimate $\mathrm{CNO}$ abundances.

\section{2. [O/Fe] Estimates: OSIRIS on SOAR}

Oxygen abundances from optical spectra are difficult to obtain, since they typically require high-resolution spectroscopy of the [O I] line at $6300 \AA$ A. However, when the carbon abundance is known, medium-resolution spectra of the $\mathrm{CO}$ features in the K-band are sufficient to esimate oxygen abundances. With the use of the Ohio State InfraRed Imager/Spectrometer (OSIRIS) on

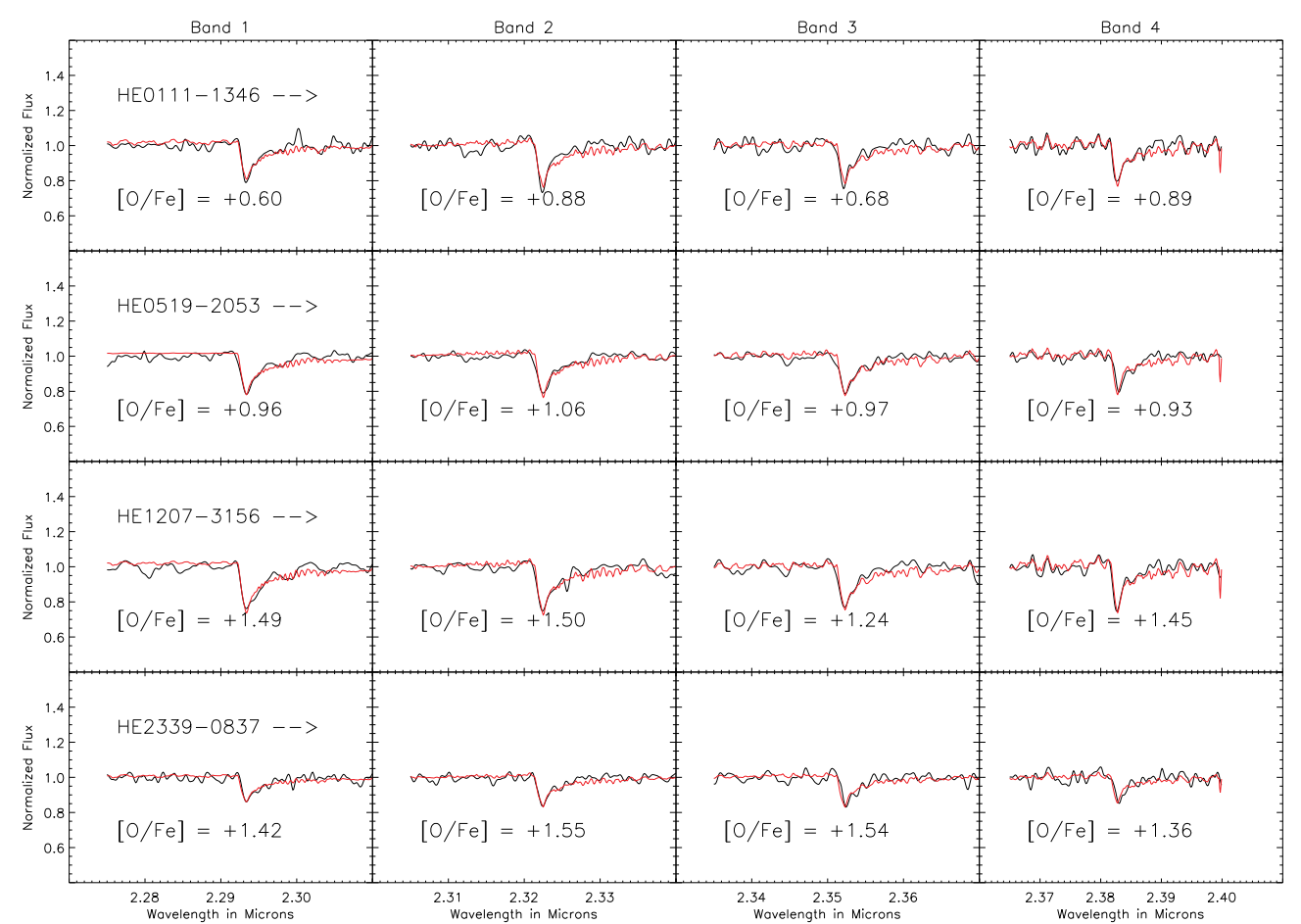

Figure 1: Estimates of $[\mathrm{O} / \mathrm{Fe}]$ for four stars. Each row shows the synthesis of the four available $\mathrm{CO}$ bands used for analysis. A robust average of the four separate estimates is taken as the value of $[\mathrm{O} / \mathrm{Fe}]$ for each star. 
the SOAR $4.1 \mathrm{~m}$ telescope, we have estimated $[\mathrm{O} / \mathrm{Fe}]$ for 57 metal-poor stars selected from the Hamburg/ESO survey (HES; Christlieb 2003; Christlieb et al. 2008), 45 of which are carbonenhanced as described by Beers \& Christlieb (2005), with $[\mathrm{C} / \mathrm{Fe}]>+1.0$. Atmospheric parameters were estimated from optical spectra (Beers et al. 2007b) as well as available optical and near-IR photometry (Beers et al. 2007a; Skrutskie et al. 2006). The spectral synthesis was achieved with use of MARCS model atmospheres (Gustafsson et al. 2008) with various values of $T_{\text {eff }}, \log g$, and $[\mathrm{Fe} / \mathrm{H}]$. Examples of the fitting of the $\mathrm{CO}$ bands in the near-IR spectra are shown in Figure 1 for four stars, where the red lines are the best-fitting synthetic spectra.

The results for all of our stars can be seen in Figure 2. Here, the results are compared to $[\mathrm{O} / \mathrm{Fe}]$ estimates that come from high-resolution spectroscopy (Masseron et al. 2010, and references therein). The different colored boxes represent the region occupied by different types of CEMP stars, and the black dashed line represents the region occupied by the carbon-normal metal poor stars of Spite et al. (2005). Our results are largely consistent with CEMP stars that have neutron-capture-element enhancements.

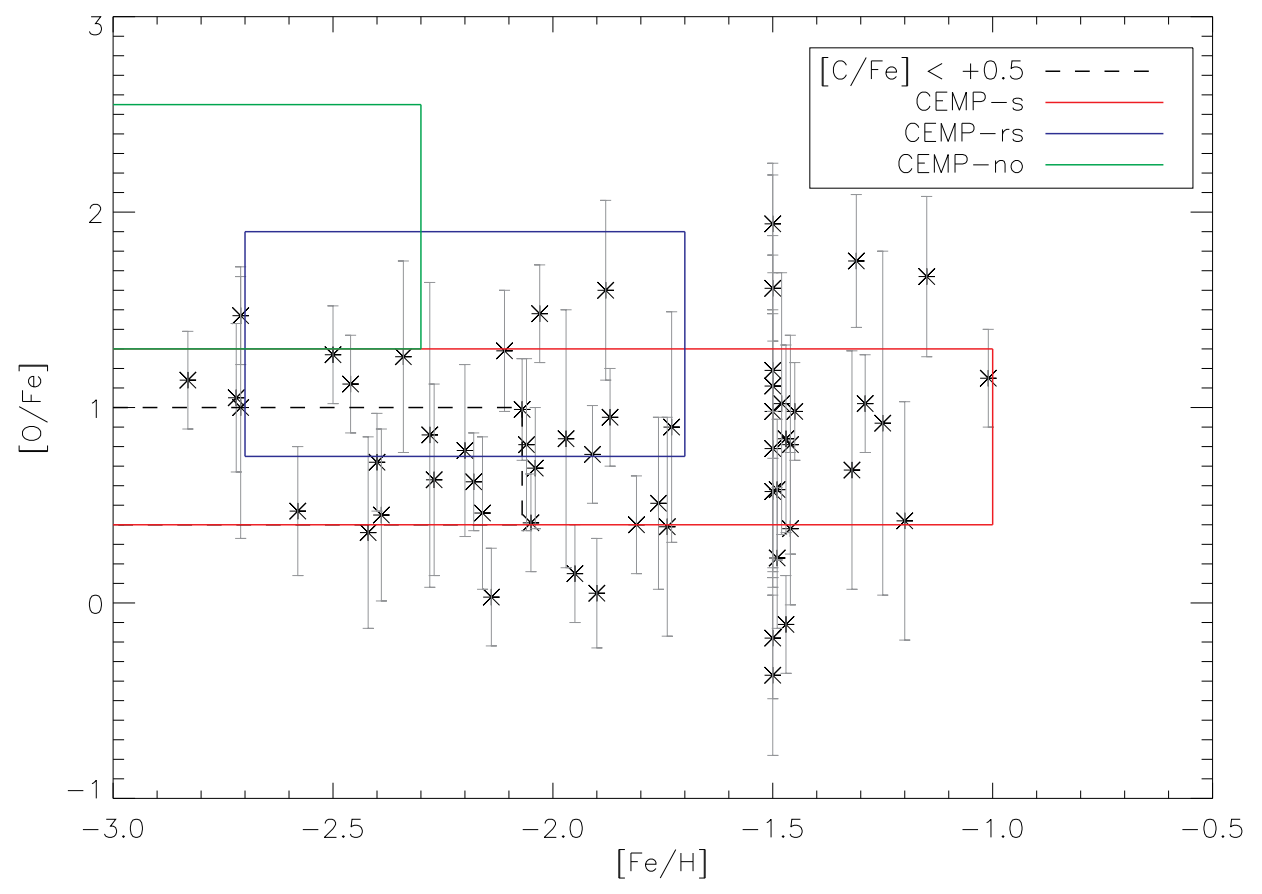

Figure 2: $[\mathrm{O} / \mathrm{Fe}]$ vs. $[\mathrm{Fe} / \mathrm{H}]$ for the entire sample. Also plotted are the regions of parameter space occupied by the different kinds of CEMP stars, based on high-resolution analysis. The region of parameter space occupied by a sample of carbon-normal metal-poor stars is also indicated.

\section{3. [C/Fe] Estimates: GMOS-S on Gemini}

We have embarked upon an effort to identify new carbon-enhanced metal-poor stars by selecting spectra from the Hamburg/ESO Survey (HES) which have a very strong CH G-band (Placco et al. 2010). This new selection is based on an new extended G-band line index which encom- 
passes the entire region from 4200 - $4400 \AA$. The first targets were observed using the Goodman High-Throughput Spectrograph on SOAR, and are presented in Placco et al. (2010). Atmospheric parameters were estimated with the NSSPP (Non-SEGUE Stellar Parameter Pipeline, developed by Y.S. Lee). The carbon abundances were estimated by an automated synthesis of the G-band (developed by T. Sivarani). The pilot program proved to be successful: $43 \%$ of stars with $[\mathrm{Fe} / \mathrm{H}]<-2.0$ were found to be carbon-enhanced. We have since continued this project with GMOS-S on Gemini. For the first sample of these targets, we found 5 of 9 with $[\mathrm{Fe} / \mathrm{H}]<-1.0$ to be carbon-enhanced. We hope to continue this survey effort and proceed with follow-up high-resolution spectroscopy of the newly discovered CEMP stars.

\section{CNO Abundances with One Instrument: XSHOOTER}

XSHOOTER is a new 3-armed spectrograph on the VLT which takes spectra in the nearUV, optical, and near-IR bands simultaneously. This enables us to estimate carbon, nitrogen, and oxygen for metal-poor stars with just one observation. We have begun a pilot program to study $\mathrm{CNO}$ abundances of metal-poor stars with this instrument. The targets were selected based on previously-determined atmospheric parameters. All of the pilot stars are metal-poor, with $[\mathrm{Fe} / \mathrm{H}]$
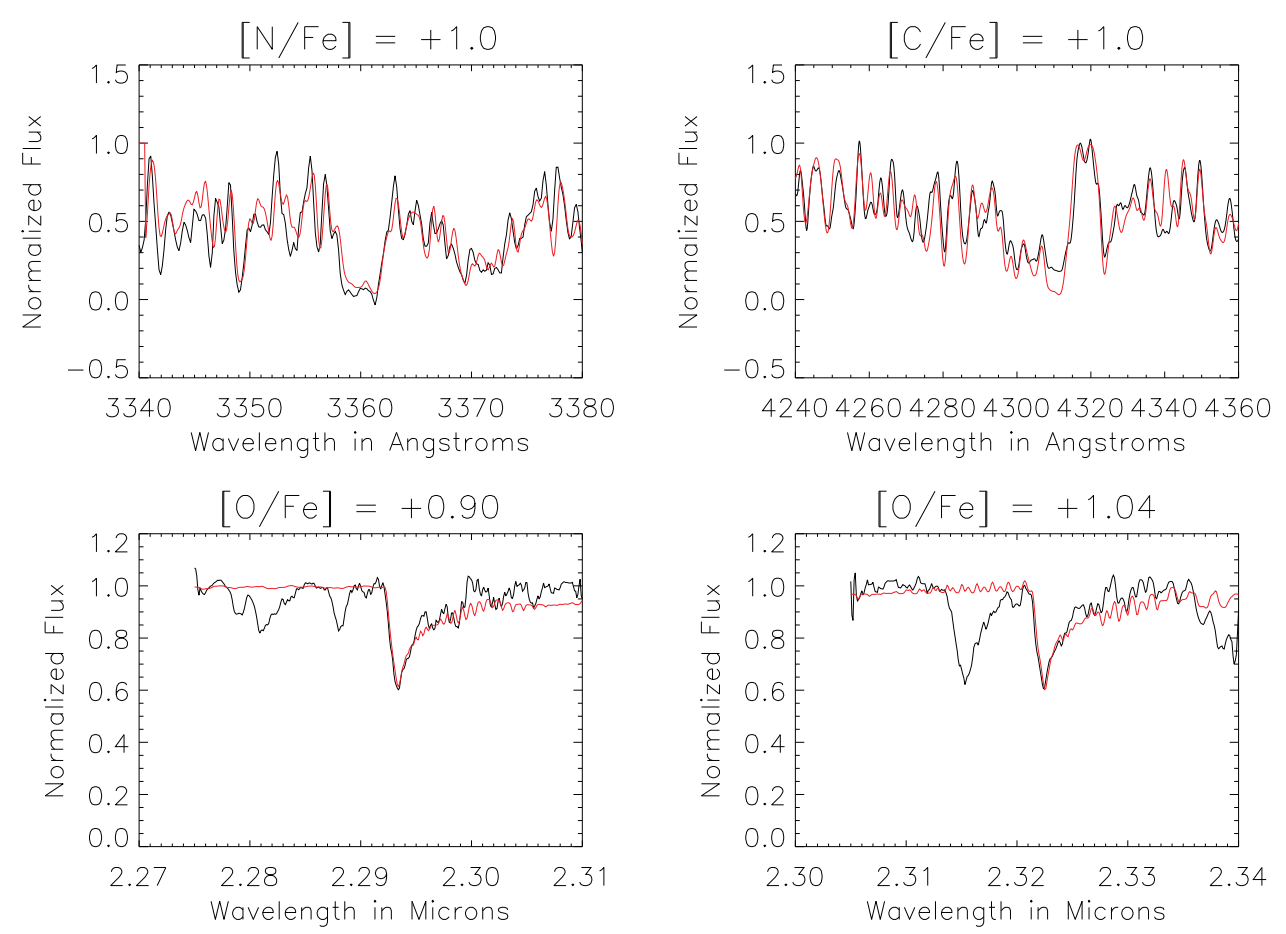

Figure 3: CNO Abundances for HE 2144-1832, observed with XSHOOTER. The features on the blue side of the fitted ${ }^{12} \mathrm{CO}$ features in the two bottom panels are ${ }^{13} \mathrm{CO}$.

$<-1.0$. It is also crucial to select targets which have low $T_{\text {eff }}(<\sim 5000 \mathrm{~K})$, as the CO bands in the near-IR spectra are not visible for higher temperatures. For the spectral synthesis, we again use MARCS model atmospheres (Gustafsson et al. 2008). Nitrogen is estimated from the NH feature at $3600 \AA$. This feature is ideal for estimated nitrogen abundances, as the $\mathrm{CN}$ bands are heavily 
dependent on carbon abundance. The carbon abundances are estimated from the $\mathrm{CH}$ G-band, and oxygen abundances are estimated from the $\mathrm{CO}$ bands as described in Section 2.

Full analysis of the sample is currently being carried out. One example of our abundance analysis is shown in Figure 3. The observations in Figure 3 are from part of the ESO VLT program 085.D-0041. Again, the red lines show the best-fitting synthetic spectra. Here, C, N, and O are estimated for HE 2144-1832 (with $[\mathrm{Fe} / \mathrm{H}]=-1.50$ ), a star which previously had no estimates of CNO. Preliminary results reveal that this star exhibits enhancements of all three elements, with $[\mathrm{N} / \mathrm{Fe}]=+1.0 \pm 0.5,[\mathrm{C} / \mathrm{Fe}]=+1.0 \pm 0.25$, and $[\mathrm{O} / \mathrm{Fe}]=+0.97 \pm 0.25$. The use of XSHOOTER provides us with the ability to make remarkable progress towards increasing the number of metalpoor stars with all three of these crucial species.

\section{Acknowledgments}

C.R.K. and T.C.B. acknowledge partial support for this work from grants AST 07-07776, PHY 02-15783 and PHY 08-22648; Physics Frontier Center/Joint Institute or Nuclear Astrophysics (JINA), awarded by the US National Science Foundation. V.M.P. and S.R. thank MSU/JINA and FAPESP for financial support.

\section{References}

Beers, T. C. \& Christlieb, N. 2005, ARA\&A, 43, 531

Beers, T. C., et al. 2007a, ApJ, 168, 128

Beers, T. C., Sivarani, T., Marsteller, B., Lee, Y. S., Rossi, S., \& Plez, B. 2007b, AJ, 133, 1193

Christlieb, N. 2003, RvMA, 16, 191

Christlieb, N., Schörck, T., Frebel, A., Beers, T. C., Wisotzki, L., \& Reimers, D. 2008, A\&A, 484, 721

Gustafsson, B., Edvardsson, B., Eriksson, K., Jørgensen, U. G., Nordlund, A., \& Plez, B. 2008, A\&A, 486, 951

Hirschi, R., Fröhlich, C. Liebendörfer, M., \& Thielemann, F.-K. 2006, RvMA, 19, 101

Masseron, T., Johnson, J. A., Plez, B., van Eck, S., Primas, F., Goriely, S., Jorissen, A. 2010, A\&A, 509, A93

Meynet, G., Ekström, S., \& Maeder, A. 2006, A\&A, 447, 623

Placco, V. M. et al. 2010, AJ, 139, 1051

Skrutskie, M. F., et al. 2006, AJ, 131, 1163

Spite, M., et al. 2005, A\&A, 430, 655 